www.ocula.it • ISSN 1724-7810 • Vol 21, No 23 (July 2020) • DOI: 10.12977/ocula2020-27

Fiori dell'anima. La simbologia dei fiori nell'immaginario religioso Flowers of the soul. The symbolism of flowers in the religious imagination a cura di Marco Papasidero e Francesco Galofaro

\title{
«Lasciate che erigano il mio letto fiorito!» Elementi vegetali nella letteratura sumerica paleo-babilonese
}

\section{Ludovica Bertolini}

Sapienza Università di Roma, IT

ludovicabertolini@hotmail.it

<https://uniroma1.academia.edu/LudovicaBertolini>

\begin{abstract}
The purpose of this article is, on the one hand, to investigate how the vegetal imaginary is used, by the scribes, to define the "other", in order to bring its inner meaning back to understandable forms. On the other hand, this work proposes an interpretation of the religious context which uses elements of flora, and human products derived from it, in the context of the cult practice connected to the couple, known from the Sumerian tradition, formed by the goddess Inanna and her husband Dumuzi.
\end{abstract}

\section{Keywords}

Flora; Inanna and Dumuzi; Abundance; Kingship; Sumerian literature

\section{Sommario/Content}

1. Introduzione

2. Alcune considerazioni sulle precedenti ricerche

3. Flora e tradizione scritta

4. Conclusioni

Bibliografia 


\section{Dcula ${ }^{23}$}

Vol 21, No 23 (July 2020) • DOI: 10.12977/ocula2020-27

Ludovica Bertolini | "Lasciate che erigano il mio letto fiorito!". Elementi vegetali nella letteratura sumerica paleo-babilonese

\section{Introduzione ${ }^{1}$}

Il mondo fenomenico, ma soprattutto la flora, costituisce un ambito di fervida riflessione per chi si approcci alla letteratura in lingua sumerica del periodo paleo-babilonese (ca. 2000-1600 a.C.). A causa di alcune lacune nella nostra conoscenza, non è sempre chiaro il contesto d'uso di questi componimenti. ${ }^{2}$ Basti accennare che i testi qui menzionati sono datati al periodo paleo-babilonese, ma si riferiscono spesso a personaggi afferenti alla cosiddetta rinascita sumerica e pertanto risalenti alla Terza Dinastia di Ur (2112-2004 a.C.).

La produzione scritta giunta fino ad oggi si costituisce come un grande insieme di testi di natura, struttura e probabilmente contesto di utilizzo differente, accomunata dall'impiego di una lingua, quella sumerica, non più in uso già dal III millennio a.C. se non in specifici ambienti e da personaggi appositamente istruiti (Rubio 2009: 11). Il materiale che verrà preso in considerazione è circoscritto a quelli che sono comunemente denominati dagli studiosi "canti d'amore" e a composizioni relative allo speciale rapporto privilegiato tra il sovrano e la dea della città di Uruk: Inanna.

Paralleli saranno poi individuati ed estrapolati da altri componimenti, tra cui inni reali e poemi di carattere epico. Il presente lavoro intende offrire una panoramica sul ruolo della sfera vegetale in tale contesto. Si mostrerà come gli scribi, attraverso l'impiego di metafore e similitudini, abbiano vivacemente attinto al mondo naturale, e nello specifico alla flora, per veicolare una serie di messaggi, stati d'animo, stimoli sensoriali, altrimenti difficili da spiegare. Inoltre, si vedrà come, nella documentazione scritta, la flora abbia un ruolo anche nella pratica rituale connessa alla sfera regale. Si tenterà quindi di dimostrare come il regno vegetale crei un ponte tra il piano letterario, quello ideologico ed infine quello religioso.

\section{Alcune considerazioni sulle precedenti ricerche}

La letteratura in lingua sumerica è spesso ricca di riferimenti a rapporti amorosi tra entità divine. Una variante del tema è costituita da una serie di composizioni che menzionano l'unione tra sovrani e divinità. In questo secondo caso, la divinità in questione è nella quasi totalità dei casi Inanna, una delle figure femminili più importanti del pantheon mesopotamico. La quantità $\mathrm{e}$ la varietà di attributi divini ad essa associati testimoniano un'evoluzione del personaggio che ha saputo attingere a numerose tradizioni tipiche di diversi centri di culto dell'alluvio mesopotamico.

1 Le abbreviazioni usate in questo articolo sono elencate all'indirizzo web della Cuneiform Digital Library Initiative(CDLI), <http://cdli.ox.ac.uk/wiki/abbreviations for_assyriology> (URL consultato in data 26 novembre 2019). Per quanto riguarda i testi cui si fa riferimento in questo lavoro possono essere consultati in traslitterazione e traduzione inglese all'indirizzo web del "Electronic text corpus of Sumerian literature (ETCSL), <http://etcsl.orinst.ox.ac.uk> (URL consultato in data 26 novembre 2019).

2 Sulla natura della letteratura in lingua sumerica, ed in generale per una panoramica sull'argomento, si veda Rubio (2009) e relativa bibliografia. 


\section{Ocula ${ }^{23}$}

Vol 21, No 23 (July 2020) • DOI: 10.12977/ocula2020-27

Ludovica Bertolini | "Lasciate che erigano il mio letto fiorito!". Elementi vegetali nella letteratura sumerica paleo-babilonese

Tra i domini che rientrano sotto la tutela di Inanna vi sono quello dell'amore sensuale, della guerra e della legittimità regale. La sua è una figura estremamente complessa e controversa, tale da aver attirato l'attenzione di numerosi studiosi (Vanstiphout 1984; Harris 19913), interessati non solo a indagare la natura intrinseca della dea, ma anche il significato di alcuni dei racconti sacri incentrati su di lei.

Ampio spazio è stato inoltre dedicato all'analisi dell'impiego di figure retoriche nelle letterature sumerica ed accadica (Wilcke 1987; Ponchia 2009), con particolare interesse al ruolo degli elementi naturali nell'ambito della costruzione dell'immaginario metaforico di queste produzioni scritte. Il valore che è stato di volta in volta associato a questi termini ed ambiti si è spesso trovato a relazionarsi con concetti quali fertilità (Couto-Ferreira 2017), potenza sessuale (Westenholz 1992: 383) e vita (Selz 2014).

Vi è però un ulteriore campo in cui la sfera vegetale compare all'interno di queste composizioni. Si tratta di atti di carattere rituale che vengono menzionati nei canti, in forme più o meno canonizzate, che dovevano richiamare alla mente del 'lettore' specifiche pratiche. ${ }^{4}$ I due momenti rituali in cui compaiono riferimenti ad erbe, fiori o piante in contesto amoroso sono quelli della preparazione della dea all'incontro con il suo futuro sposo e dell'allestimento del letto sacro. Di seguito verranno presentate le specie individuate nei canti, ${ }^{5}$ con le relative attestazioni.

\section{Flora e tradizione scritta}

\subsection{Girin}

Il termine sumerico girin racchiude una serie di significati che variano dal campo semantico della purezza a quello della flora. ${ }^{6}$ Generalmente, nel secondo caso, il termine viene tradotto come 'fiore', ma può riferirsi a un albero o a un frutto, ed è preceduto dal segno ĜIŠ che assume il valore di determinativo

3 La bibliografia inerente alla figura di Inanna è molto ampia. Si è scelto qui di dare una panoramica generale dei lavori noti. Per ulteriori approfondimenti si veda la bibliografia degli articoli indicati.

4 Ritengo che le pratiche descritte nei componimenti indagati dovessero essere ben conosciuti dallo scriba $\mathrm{o}$, in generale, da chi si relazionava in qualunque modo con il testo (non si intende qui discutere sulla presunta natura orale di alcune di queste composizioni per cui si rimanda ad Alster 1972). Questo sembrerebbe potersi ipotizzare sia in virtù del fatto che queste azioni vengono descritte in maniera standardizzata, sia perché non vengono mai indagate in maniera approfondita, come se appunto fosse sufficiente accennare ad alcune fasi del rituale per richiamare alla mente la sua funzione e i particolari del suo svolgimento o preparazione.

5 Nella selezione si è deciso di non prendere in considerazione elementi vegetali già ampiamente analizzati in altre pubblicazioni (si vedano a tal proposito CoutoFerreira 2017 e Zisa in stampa).

6 Sefati (1998: 174) ritiene che si tratti di un aggettivo che qualifica il nome precedente e dà una traduzione del termine come "greenery" in base al contesto. 


\section{Dcula ${ }^{23}$}

Vol 21, No 23 (July 2020) • DOI: 10.12977/ocula2020-27

Ludovica Bertolini | "Lasciate che erigano il mio letto fiorito!". Elementi vegetali nella letteratura sumerica paleo-babilonese

per elementi della classe vegetale. ${ }^{7}$ Nei testi analizzati, il termine compare in sole quattro composizioni, appartenenti al cosiddetto Ciclo di Inanna e Dumuzi. ${ }^{8}$

In $D I F$, il termine è utilizzato in principio di quella che sembra essere la descrizione di un paesaggio rigoglioso, immerso nei pascoli, al di fuori della città (DI F ll. 1-2). Il complesso campo semantico viene delineato dalla presenza di alcuni elementi che afferiscono a tale ambito, tra cui i personaggi del pastore (emesal: ${ }^{9}$ suba; sumerico: sipad) e dell'agricoltore (emesal: mungar; sumerico: engar), noti non solo come rappresentanti di queste aree rurali, ma anche come personaggi ricorrenti in composizioni di tema amoroso. Il termine girin può essere qui tradotto come 'fiore/i' $\mathrm{i}^{10} \mathrm{O}$ 'prato fiorito' ed è l'oggetto della meraviglia della dea, la quale, in linea con il tono erotico del testo, si augura di incontrare lì il suo amato. In questo caso è possibile cogliere una differenziazione tra il livello letterale e quello metaforico (Couto-Ferreira 2017; Zisa in stampa ${ }^{11}$ ). Personalmente ritengo che girin sia in tal caso impiegato per alludere al giovane amato e non alla dea Inanna, poiché viene esplicitamente detto che la dea posa il suo sguardo su di esso, facendo pertanto pensare che ci si stia riferendo ad un luogo che è estraneo alla sua persona. Se da una parte è vero che spesso termini che indicano campi o giardini possono nascondere riferimenti al corpo femminile, e specialmente ai suoi organi riproduttivi, si deve tuttavia ammettere che il medesimo campo semantico si adatta contestualmente anche alla sessualità maschile. Estranei non sono, infatti, i riferimenti ad alberi e piante di vario genere che, se in alcuni passi alludono metaforicamente al corpo dell'amato, in altri, riportano la scena ad un'ambientazione - quella della steppa o del deserto - che simbolicamente lo richiama. In questo caso, è evidente come, alle linee iniziali del canto, ad un primo livello interpretativo si stia facendo riferimento al paesaggio in cui la scena è ambientata. Tuttavia, ad un secondo livello, si percepisce un legame tra l'elemento vegetale e il dio Dumuzi:

7 Il determinativo in sumerico è un dispositivo che, precedendo un nome, ne indica la classe semantica di appartenenza; nel caso del segno ĜIŠ esso è associato in genere a nomi di piante e manufatti in legno, di qualsiasi dimensione. Nella trascrizione i determinativi sono posti in apice davanti al nome che devono classificare, ma, più raramente, possono anche seguirlo.

8 Per un'edizione aggiornata di questi componimenti si veda Sefati (1998). I testi da qui in poi denominati con la sigla DI (seguita dalla lettera che identifica il singolo brano della collezione) si riferiscono al summenzionato gruppo di opere incentrate sulle vicende della coppia divina di Dumuzi ed Inanna.

9 Il registro emesal è considerato un dialetto o una forma particolare del sumerico che presenta grafie diverse dal dialetto originale, è in generale collegato a discorsi pronunciati da figure femminili, ma è anche caratteristico di alcune composizioni che rientrano nel repertorio del sacerdote lamentatore conosciuto come 'gala'. In merito al registro emesal si vedano Schretter (1990); Whittaker (2002).

10 Si noti come il sumerico non esprima sempre il plurale.

11 Ringrazio il Dott. Zisa per avermi dato accesso in anticipo al manoscritto del suo articolo. 


\section{Ocula ${ }^{23}$}

Vol 21, No 23 (July 2020) • DOI: 10.12977/ocula2020-27

Ludovica Bertolini | "Lasciate che erigano il mio letto fiorito!". Elementi vegetali nella letteratura sumerica paleo-babilonese

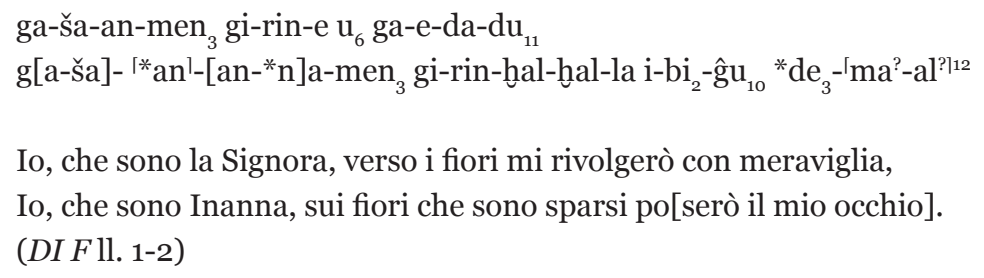

L'espressione i-bi ${ }_{2}-\hat{g}_{10} \mathrm{de}_{3}-\mathrm{Ima}^{\text {? }}$-al ${ }^{\text {? }}$, forma emesal, ricalca quasi alla perfezione l'enunciato della stessa Inanna in DI P (ll. 22-23), questa volta utilizzato per indicare come la dea abbia scelto il suo amato tra le genti del Paese per innalzarlo al ruolo di loro dio:

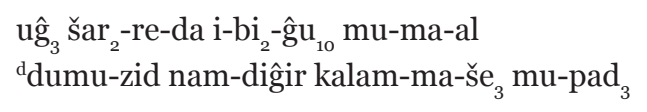

La finzione metaforica continua anche alle linee successive di $D I F$, dove la dea Inanna accenna alla propria intenzione di correre al girin e di sdraiarsi sopra di esso (ll. 5-6). Poiché in questi testi non è affatto strano incontrare velati o diretti riferimenti al rapporto sessuale tra due amanti, sarebbe ragionevole aspettarsi che l'espressione gi-rin-e da-nu ${ }_{2}$ sia usata in maniera eufemistica per riferirsi al desiderio della dea di unirsi a Dumuzi. Anche in questo caso, la lettura del passo rivestirebbe una doppia valenza: da una parte, la distesa di fiori fa da sfondo all'azione, costituendone l'elemento principale nella scena, dall'altra, allude alle caratteristiche tipiche dell'amato. Il fatto che, nel caso di $D I F$, dietro a girin si debba ricercare un attributo dell'elemento maschile di questa coppia, ritengo che sia avvalorato dalla presenza di una metafora alla linea 26: qui, nonostante l'incapacità di ricostruire i primi due segni, si può ragionevolmente riconoscere un passo del discorso di lode intonato da Inanna in onore del suo amato, nel quale ella paragona l'attrattiva di Dumuzi (hili) ad una distesa di fiori. Analogamente, attraverso una vera e propria similitudine, la dea accosta la desiderabilità del dio a quella dei fiori: gi-rin-na-gin ${ }_{7}$ hi-lizu dug ${ }_{3}$-ga-am ${ }_{3}$. Il testo in questione, come si vedrà oltre, racchiude una moltitudine di espressioni metaforiche o simboliche che concorrono alla delineazione del carattere e dell'aspetto dell'amato. Pertanto è ragionevole prendere in considerazione il fatto che in girin si nasconda un'allusione al giovane dio.

Similmente, in contesto amoroso, girin è associato a due riferimenti più diretti del rapporto consumato tra i due dei. Nel testo DI $T$, in merito alla

12 Per la ricostruzione della frase si veda fonte CBS 4589 l.2 in Sefati (1998: 171).

13 Nel sistema numerico mesopotamico, a base sessagesimale, il numero tremilaseicento e suoi multipli vengono spesso utilizzati, in letteratura, per indicare moltitudine o totalità del sostantivo cui sono associati. 


\section{Deula ${ }^{23}$}

Vol 21, No 23 (July 2020) • DOI: 10.12977/ocula2020-27

Ludovica Bertolini | "Lasciate che erigano il mio letto fiorito!". Elementi vegetali nella letteratura sumerica paleo-babilonese

preparazione della cella della dea per l'arrivo del futuro sposo, il letto su cui la sacra unione avrà luogo viene cosparso di fiori:

ma $\hat{g}_{6}-$ par $_{4}-$ ra-ĝu $_{10}-$ de de $_{3}$-ma-ab-BU-ne

gišr-nu ${ }_{2}$ gi ${ }_{4}$-rin-na- $\mathrm{gu}_{10} \mathrm{de}_{3}{ }^{*}-\mathrm{ma}^{*}$-ab-gub-bu-ne

Che essi erigano!14 ${ }^{14}$ per me nella mia casa del gipar,

Che essi erigano per me il mio letto di fiori!

(DI T ll. 39-40)

La cella del tempio, con il letto ricoperto di erbe o fiori, ricorda il prato che la dea osservava con meraviglia in $D I F$. Anche in questo caso, inoltre, Inanna si accinge ad unirsi con il suo amato in un luogo puro, connotato dalla presenza di elementi naturali, anche se estrapolati dal loro normale contesto per essere impiegati in un'occasione dai chiari connotati rituali. Stessa scena compare anche in $D I D 1$, dove il giaciglio è ricoperto di fiori e lapislazzuli:

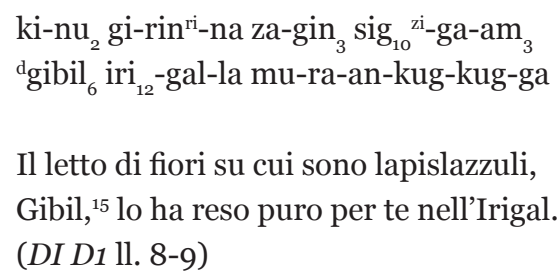

Il lapislazzulo compare in associazione con il letto di entità divine anche in altre occasioni (DI T l. 41; Ur-Namma A 1. 159 ETCSL 2.4.1.1; Cilindri di Gudea ll. 1017, 1200 ETCSL 2.1.7) e sempre in quello che potrebbe definirsi un ambito rituale. Non è chiaro in che rapporto dovessero essere le pietre e l'elemento vegetale, se si faccia riferimento con questo accostamento a particolari proprietà condivise da entrambi, o se il za-gin ${ }_{3}$ serva da termine di paragone nella descrizione del primo.

Ultimo testo analizzato per presentare il termine girin è $D I Z$ (ll. 17'-19'). Ancora una volta si incontra la dea Inanna desiderosa di poter abbracciare Dumuzi e di poter godere della sua compagnia sul giaciglio sacro, dopo che il giovane sarà stato benedetto da Enlil e Suen: ${ }^{16}$

14 La radice verbale in questione non è conosciuta, il segno corrispondente è scritto in lettere maiuscole per indicare che, nonostante esso sia ben visibile nel testo, non si è sicuri del valore da attribuirvi, la traduzione è pertanto basata sul contesto (cfr. Jacobsen 1975: 79-80).

15 Gibil è una figura divina del pantheon mesopotamico connessa all'elemento del fuoco, nel testo in questione si afferma che egli sia il responsabile dei rituali relativi alla nam-ga-ša-an-e 'dell'essere signora', dove la particella nam- serve a creare la forma astratta di un sostantivo.

16 Si tratta rispettivamente del dio poliade di Nippur, considerato una delle maggiori personalità divine del pantheon sumerico, e del dio luna della città di Ur, padre della stessa Inanna. 


\section{Ocula ${ }^{23}$}

Vol 21, No 23 (July 2020) • DOI: 10.12977/ocula2020-27

Ludovica Bertolini | "Lasciate che erigano il mio letto fiorito!". Elementi vegetali nella letteratura sumerica paleo-babilonese

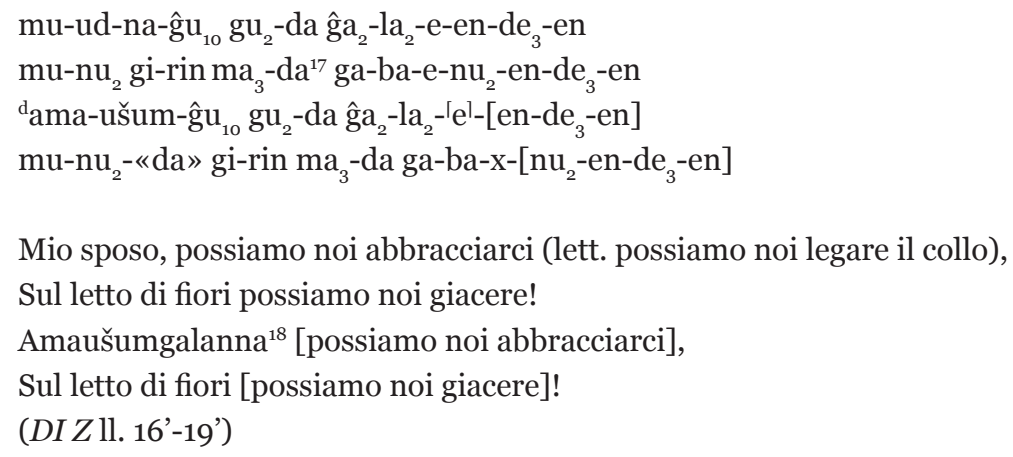

Quando usato in associazione al letto sacro di una divinità il termine girin sembra porre l'accento sulla situazione e sull'ambientazione che fa da sfondo alla scena, piuttosto che sui personaggi coinvolti. Quando invece compare da solo, come in $D I F$, esso parrebbe prediligere un livello interpretativo più strettamente legato alla definizione del carattere dell'amato.

In forte assonanza con girin è il termine gurun (lett. frutto; fiore; attrattiva sessuale; figurina) che, come si può notare, ricopre lo stesso spettro semantico:

ĝišhašhur a $\hat{g}_{2}-s a \hat{g}-\hat{g}_{2}$ gurun il - -la- $\hat{g}_{10}$ hi-iz ${ }^{\text {sar }}-\mathrm{am}_{3}$ a ba-an- $-\mathrm{du}_{11}{ }^{19}$

Il mio prezioso melo che porta frutti, è lattuga che egli/ella ha innaffiato.

(DIE 1. 4)

Nell'esempio appena citato gurun è funzionale alla descrizione del personaggio maschile ${ }^{20}$ assieme a hašhur e hiz. Che la frase sia pronunciata dalla dea Inanna è chiaro per la presenza del segno a $\hat{g}_{2}$, forma emesal di nig $\hat{g}_{2}$ 'cosa'; inoltre, la menzione dell'albero sembra essere un rimando alla figura dell'amato. Gli alberi, infatti, appaiono spesso in relazione a figure maschili. In alcuni inni reali, il potere del sovrano è dipinto come un albero che unisce cielo e terra, il quale con i suoi rami si estende sopra le genti, proteggendole come una chioma. Il sostantivo gurun, invece, serve a veicolare l'idea di benessere e abbondanza che l'amato è in grado di garantire con la propria presenza e le proprie cure.

17 Per la costruzione grammaticale usata alle linee 17' e 19' della fonte Ni 4552, si veda il commento in Sefati (1998:283-285), in particolare quello relativo alle linee 3-6, con relativo rimando a $D I G 1.15$.

18 Nome di divinità che attorno al 2500 a.C. viene a convergere con la figura di Dumuzi. Al momento della stesura di questi testi i due personaggi sono ormai totalmente sovrapponibili.

19 La linea è stata ricostruita sulla base delle fonti AO 5385, Ni 9846, IM 11087.

20 In favore di questa associazione Sefati (1998: 167), mentre Zisa (in stampa) propone alternativamente che il termine possa riferirsi alla stessa Inanna in base all'associazione tra 'lattuga' e 'vulva' in contrapposizione a quella tra 'acqua' e 'liquido seminale maschile'. Entrambe le letture, seppure antitetiche, sono ugualmente supportate grammaticalmente. 


\section{Dcula ${ }^{23}$}

Vol 21, No 23 (July 2020) • DOI: 10.12977/ocula2020-27

Ludovica Bertolini | "Lasciate che erigano il mio letto fiorito!". Elementi vegetali nella letteratura sumerica paleo-babilonese

Una frase molto somigliante compare in $D I B$ alla linea 29. Si tratta di un dialogo d'amore tra i due amanti, durante il quale la dea fa promettere al giovane di esserle fedele. La presenza di termini in registro emesal (ll. 13-33), lascia intendere che la seconda parte del dialogo contenga un unico e continuo intervento della dea, pertanto si potrebbe immaginare che la persona cui si sta rivolgendo sia proprio l'amato. ${ }^{21}$ Se si accetta di vedere Dumuzi nell'oggetto del discorso di Inanna, allora è probabile che, in questo caso,

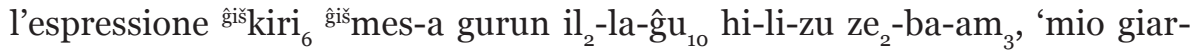
dino di alberi mes che porta frutto, la tua attrattiva è dolce' (1.29) serva a caratterizzare l'aspetto di desiderabilità che contraddistingue il dio agli occhi della consorte.

Con lo stesso intento, gurun viene impiegato anche nella definizione dell'ambiente creato da Enki per il proprio tempio in Il viaggio di Enki a Nippur (ETCSL 1.1.4); qui il santuario del dio appare come una montagna che galleggia tra i canneti. Le acque che lo circondano sono piene di pesci che nuotano scuotendo le onde con il loro fare frenetico, mentre gli uccelli covano nei frutteti verdi carichi di frutta (1l. 75-82). È chiaro che l'immagine che si vuole dare di questo sito è quella di un luogo perfetto, dove abbonda tutto ciò che è necessario alla vita. La presenza del tempio, come immagine della tutela divina sulla città di Eridu, produce un diffuso senso di prosperità veicolato dalle immagini di animali e vegetazione che si moltiplicano e prosperano per volere della divinità. Lo stesso avviene per la dea e per il suo Paese grazie all'amore del futuro sposo di questa, il quale rappresenta a sua volta l'immagine prototipica del sovrano giusto e attento che, prendendosi cura della divinità, fa sì che essa conceda il suo favore al Paese intero. Per il medesimo motivo l'amorevole cura di Inanna per il mitico sovrano di Uruk, Enmerkar, in Enmerkar ed il signore di Aratta (ETCSL 1.8.2.3), permette al tempio della dea, situato nell'area sacra di Kullaba, di risplendere come un albero mes che porta frutti (ETCSL 1.8.2.3 1l. 25, 216, 266).

Desiderosi di acquisire, mantenere e ostentare, un tale favore sono sicuramente anche i sovrani della Terza Dinastia di Ur. Šulgi, secondo sovrano della dinastia, in un inno reale viene descritto esattamente come un albero da frutto rigoglioso, attestando così che la sua fortuna è legittimata dall'intervento divino che gli garantisce un regno prospero (Šulgi D l. 33 ETCSL 2.4.2.04). $\mathrm{Nel}$ caso, invece, di Samsuiluna di Babilonia il termine gurun è impiegato in una benedizione rivolta al sovrano con l'intento di rendere la sua vita lunga (ETCSL 2.8.3.5 l. 45), apparentemente spostando il focus dal Paese alla figura del sovrano.

Tuttavia, immagini di luoghi floridi possono anche essere impiegate in letteratura con intenti diametralmente opposti. Nel mito Inanna ed Ebih (ETCSL 1.3.2), la dea di Uruk ingaggia una lotta contro la montagna Ebih, colpevole di non averle presentato adeguatamente omaggio; una delle imprudenze della

21 Il problema nasce dal fatto che nel testo non vi è un riferimento diretto al giovane dio. Ad essere menzionata è la dea Dumuzi-abzu (1. 30), che nonostante l'omofonia con lo sposo di Inanna, nel III millennio è conosciuta come una divinità femminile e solo in periodi più tardi compare come figura maschile (George 2003: 861). 


\section{Dcula ${ }^{23}$}

Vol 21, No 23 (July 2020) • DOI: 10.12977/ocula2020-27

Ludovica Bertolini | "Lasciate che erigano il mio letto fiorito!". Elementi vegetali nella letteratura sumerica paleo-babilonese

montagna è proprio quella di ostentare una eccessiva magnificenza e di credersi al livello stesso degli dei. Inanna, in risposta, si scaglia con tutti i suoi poteri contro le pendici rigogliose di Ebih per distruggerle e raderle al suolo.

\subsection{Hašhur}

Si è avuto modo già di incontrare il termine hašhur 'mela' o 'melo'. È abbastanza frequente nel contesto dei componimenti amorosi e spesso può capitare in combinazione con il termine kiri ${ }_{6}{ }^{22}$ Hašhur viene menzionato in quattro inni reali dedicati al sovrano Šulgi ed è nella maggior parte dai casi utilizzato per descrivere indirettamente qualità del re insieme $\mathrm{o}$, in contrapposizione, al cedro (ş̌šerin). Nell'inno Šulgi P (ETCSL 2.4.2.16) ad esempio, egli viene definito da Lugalbanda come un cedro irrigato che si erge tra i meli:

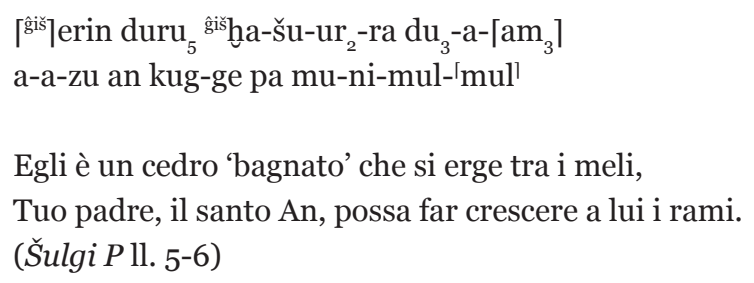

Seppur non sia ben chiara quale sia la caratteristica che lo contraddistingua, poiché dal testo non si può estrapolare con certezza cosa nell'immaginario sumerico il melo avesse in meno rispetto al cedro, l'espediente retorico deve servire a far risaltare degli aspetti del re. In realtà, sia il melo che il cedro, nella letteratura in lingua sumerica, sembrano alberi da frutto molto apprezzati per i loro prodotti. Tuttavia, ciò che può differenziare le due specie arboree in questo particolare esempio è l'aggiunta dell'aggettivo duru ${ }_{5}$, 'bagnato' o 'irrigato'. La mia impressione è che il passo in questione in Šulgi $P$ intenda solo sottolineare che il sovrano è un cedro più rigoglioso in un frutteto già di per sé benedetto dal favore divino. Egli spicca con i suoi rami che si estendono, grazie all'aiuto del dio An, in un Paese florido e prospero. Un'espressione simile è contenuta alla linea 35 di Šulgi $D$ che si vedrà nel paragrafo dedicato al cedro.

Nell'inno reale Šulgi $N$ (ETCSL 2.4.2.14), alla linea 7 del testo, un gruppo di meli splendenti posti a ridosso del canale costituisce il luogo in cui il sovrano potrà incontrare la sua compagna e il figlio avuto da lei. Nel caso specifico di questo esempio, l'aspetto erotico di solito associato a frutteti e corsi d'acqua ${ }^{23}$

22 Con il sostantivo kiri ${ }_{6}$ ci si riferisce ad un'area caratterizzata dalla presenza di alberi o piante generalmente coltivate. Quando non specificato altrimenti, il kiri ${ }_{6}$ parrebbe essere un giardino di palme da dattero (Greco 2015: 21). Va inoltre considerato che un palmeto spesso ospita una moltitudine di specie vegetali grazie alla presenza delle lunghe foglie in grado di procurare l'ombra necessaria alla semina di esemplari più delicati.

23 Per il ruolo giocato da canali e corsi d'acqua nelle vicende d'amore divine, si vedano composizioni quali Enki e Ninhursaga (ETCSL 1.1.1), Enlil e Ninlil (ETCSL 1.2.1); entrambi i testi sono trattati da Leick (1994). Per il rapporto tra irrigazione e sessualità si veda Cooper (1989). 


\section{Ocula ${ }^{23}$}

Vol 21, No 23 (July 2020) • DOI: 10.12977/ocula2020-27

Ludovica Bertolini | "Lasciate che erigano il mio letto fiorito!". Elementi vegetali nella letteratura sumerica paleo-babilonese

perde la sua valenza sessuale per divenire un luogo dove il sovrano potrà trascorrere piacevolmente il suo tempo con gli affetti familiari. Questo non sembra però il caso dell'inno Šulgi $Z$ (ETCSL 2.4.2.26), dove i meli assieme ad altri alberi da frutta accolgono l'incontro tra il re e la dea. Una tale differenza di utilizzo del termine fa ritenere che l'idea di fondo che gli scribi volessero richiamare, sia quella della piacevolezza e della dimensione privata di ciò che può avvenire in questi frutteti. Le fronde degli alberi da una parte offrono una gradevole protezione dal sole, dall'altra un certo riparo da occhi indiscreti. Così facendo allontanano la scena dalla vita frenetica della città per riportare l'uomo ad una dimensione quasi atemporale. In più, i giardini e gli orti costituiscono un punto di incontro tra l'ordinamento del cosmo, che ha il suo culmine nella città, e gli spazi esterni della steppa, soggetti a differenti leggi e tutele - naturali e divine - più vicine ad uno stato selvaggio pre-civile. Il frutteto è pertanto una forma di caos ordinato in cui l'uomo è in stretto rapporto più con i sensi che con la ragione (si veda Besnier 2002 sul ruolo ambiguo del giardiniere). Anche il riferimento costante alla presenza di frutti, alla dolcezza dei prodotti della terra, alla rigogliosità delle erbe piantate ed irrigate, sono tutti chiari richiami ad una sensualità che nel mondo vegetale trova così modo di esprimere una serie di aspetti prettamente umani come l'eccitazione sessuale, l'innamoramento, l'intraprendenza di un giovane amante, la maturità sessuale ecc.

Nel canto $D I B$, Inanna ${ }^{24}$ si riferisce al giovane amato con tali parole: ${ }^{25}$

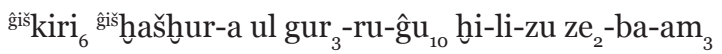

ĝ̀škiri ${ }_{6}$ ĝišmes-a gurun il ${ }_{2}-$ la-ĝ $_{10}$ hi-li-zu ze ${ }_{2}-$ ba-am $_{3}$

Mio giardino di meli che porta gioia, la tua attrattiva è dolce,

Mio giardino di alberi mes che portano frutto, la tua attrattiva è dolce.

(DI B 11.28-29)

È chiaro come la metafora del giardino di meli e mes, nell'ottica di Inanna, serva a richiamare la floridezza, l'attrattiva e la dolcezza del giovane amato. A favore dell'attribuzione delle due metafore sopra citate al dio Dumuzi, potrebbe concorrere l'augurio della dea Inanna in $D I Q^{26}$ (ll.7-9, 13-15, 19-21):

24 I nomi di Inanna o Dumuzi non compaiono mai chiaramente in questo caso; uniche eccezioni solo la menzione della dea nella dossologia finale, che farebbe propendere per identificare la fanciulla del testo con Inanna, e quella di Dumuzi-abzu, che nel contesto sembra essere stata scambiata dallo scriba con il Dumuzi di Badtibira, noto sposo della Signora di Uruk.

25 È la donna a parlare, visto l'uso del registro emesal. Questa si rivolge all'amato chiamandolo prima 'giardino', poi 'figurina' $\left(\operatorname{dim}_{3}\right)$ - forse una statuetta - ornata di pietre preziose. Sebbene nelle linee precedenti l'uomo avesse elencato le attrattive della giovane, menzionando anche piante, tra cui l'orzo (še), non vi è concordanza tra le metafore nelle due sezioni del canto. Inoltre, è nota l'associazione tra l'albero mes, come si vedrà oltre, e lo sposo della dea Inanna. Per queste ragioni propendo per identificare i due personaggi con Inanna e Dumuzi e attribuisco alla prima il passaggio alle linee 28-29.

26 Il presente componimento è trattato da Sefati e Klein (2012: 311-314). 


\section{Ocula ${ }^{23}$}

Vol 21, No 23 (July 2020) • DOI: 10.12977/ocula2020-27

Ludovica Bertolini | "Lasciate che erigano il mio letto fiorito!". Elementi vegetali nella letteratura sumerica paleo-babilonese

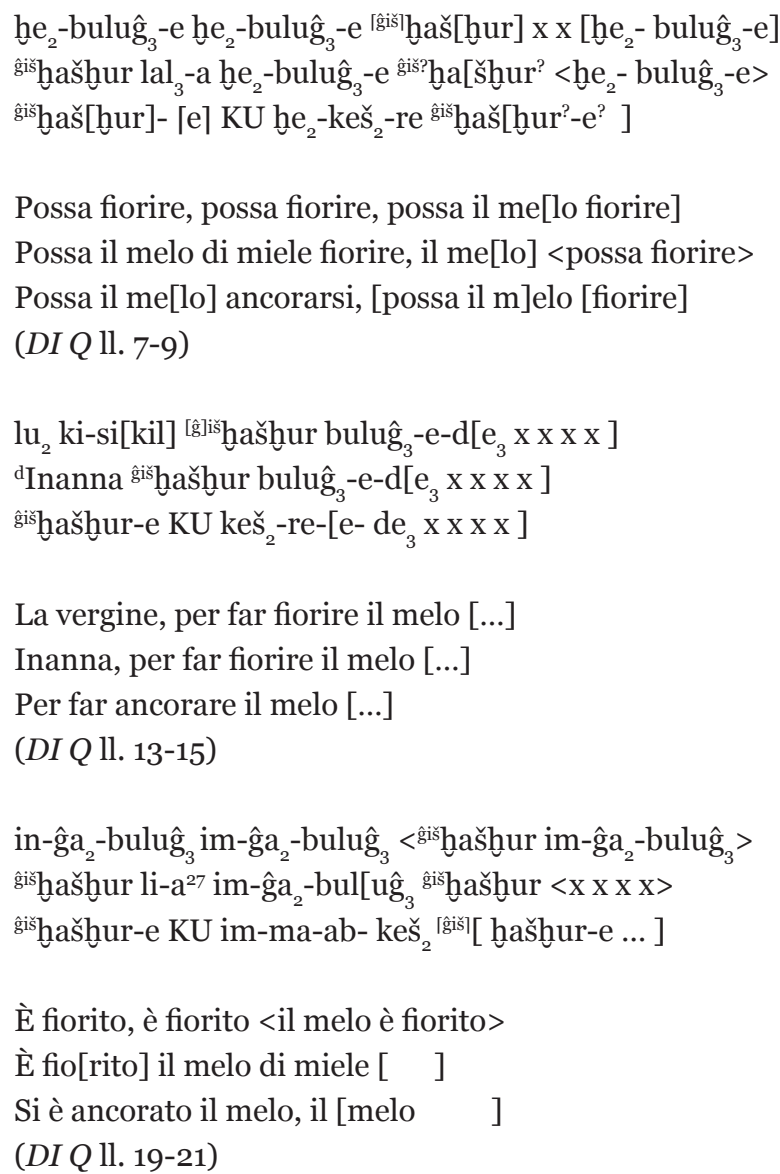

Sebbene sia difficile riuscire a capire se la metafora si riferisca al giovane o alla sua amata, ritengo che si potrebbe ugualmente tentare di ricollegare il melo al dio Dumuzi. Alle linee 4-6, infatti, la dea riporta di aver ricevuto dei doni dal toro selvaggio (epiteto di Dumuzi), il quale si sarebbe recato all'abitazione della dea con dei presenti. A questo punto Inanna esprime il desiderio che alcune piante (melo, rapa, pioppo e vite) possano crescere forti, germogliare, dare frutti e fissare saldamente le loro radici nel terreno. Ritengo pertanto che in questo caso si sia in presenza di una benedizione per l'amato. Egli verrebbe qui rappresentato attraverso la metafora vegetale, coerente con l'offerta di doni che il dio ha presentato al tempio della sua sposa. D'altronde ben noti sono passi di questo genere in cui il giovane amato viene benedetto dalla dea con auguri di lunga vita, regno saldo e prosperità. Va però anche detto che la proposta di identificare Dumuzi con le piante citate nel canto potrebbe essere supportata anche da alcune somiglianze con dei passi di $D I F$ in cui la dea, come si vedrà nel prossimo paragrafo, fa libagioni di acqua per far 'crescere' - probabilmente si deve intendere nel senso di 'prosperare' anche se sono ovviamente attivi contemporaneamente vari livelli interpretativi - il suo albero mes nel quale è espressamente da vedersi il personaggio di Dumuzi.

27 Si veda il commento di Sefati e Klein (2012: 317) alla linea 20 del testo in analisi. 


\section{Dcula ${ }^{23}$}

Vol 21, No 23 (July 2020) • DOI: 10.12977/ocula2020-27

Ludovica Bertolini | "Lasciate che erigano il mio letto fiorito!". Elementi vegetali nella letteratura sumerica paleo-babilonese

\subsection{Mes}

L'albero mes, purtroppo non ancora identificato, è ben noto nella letteratura in lingua sumerica di questo periodo. Questo albero sembra rivestire una certa importanza sia per il suo valore in ambito regale, sia per la meraviglia che doveva suscitare in chi lo guardasse. Gli sono spesso accostati appellativi come bianco o splendente (babbar ${ }_{2}$ ), vitale (zid), eccelso (mah), grande (gal).

Il fondatore della Terza Dinastia di Ur, Ur-Namma, viene definito "albero mes bianco che cresce in un luogo piacevole ad Uruk" (lugal mes babbar urim $_{5}{ }^{\mathrm{ki}}$-ma ki dug $\mathrm{g}_{3}$-ge $\mathrm{mu}_{2}$-a). Purtroppo esistono solo due attestazioni in tutta la letteratura di un albero mes bianco (Ur-Namma I ii 8 ETCSL 2.4.1.a; Enmerkar e il signore di Aratta 1.27 in questo caso però è utilizzato come vehicle in una metafora riguardante il tempio fatto costruire dal re Enmerkar), e non è possibile capire se costituisca una specie diversa o se questa sua proprietà serva ad evidenziare una qualche qualità specifica del tenor. È ad ogni modo possibile immaginare che l'effetto che si voglia ricercare grazie a queste figure retoriche sia di riproporre la stato di meraviglia che persone o cose associate a tale albero potessero infondere in chi si trovasse al loro cospetto. Šulgi, successore di Ur-Namma, nel suo inno Šulgi $D$, viene rappresentato come un albero mes vitale (o 'retto', dato il doppio valore del termine zid), carico di frutti, che ispira ammirazione. Questo secondo esempio sembra avvalorare quanto detto per il testo precedente: il mes suscita stupore per la sua bellezza, la sua ricchezza ed abbondanza di frutti. Alla linea 56 dello stesso testo, il re è di nuovo paragonato a questa splendida pianta e si asserisce in tale occasione che Enki lo abbia favorito come un albero mes e una palma da dattero (ŝiš ĝišnimbar). Per lui An ha decretato un destino favorevole, mentre Enlil gli ha concesso la signoria sul Paese. Šulgi, in questo modo appare come un motivo di orgoglio e ammirazione anche per le divinità del pantheon, che gli rivolgono le proprie attenzioni e lo tutelano nella sua ascesa. Si comincia a vedere già in questo esempio un'ulteriore caratteristica di personaggi così vicini alla sfera divina come i sovrani, mitici o storici, ossia la loro capacità di entrare in contatto con piani diversi. Tale aspetto ben si appresta a venir rappresentato dall'immagine dell'albero, richiamando l'immagine dell'axis mundi. Tale concetto diviene in parte più esplicito in Šulgi $P$, nelle parole della dea Ninsumun, la quale, riferendosi a Šulgi nell'ambito di un dialogo con il mitico re di Uruk Lugalbanda, lo descrive in tali termini:

mes pa mul-ĝu ${ }_{10}$-um ki ma-an-dar?

[x] 'mal-dam 'mul-mu ma-a-tum ${ }_{2}$

$[\mathrm{x}]$ X MUŠ $\times$ MUŠ! $\left(\right.$ ERINA $\left._{8}\right)-\hat{g}_{10}$-um ur ${ }_{2}$ ma-la-gurl

Egli è il mio albero mes dagli ampi rami, apre il terreno per me,

[x] negli anni porta abbondanza per me,

Egli è il mio $[\mathrm{x}]$ nardo,${ }^{28}$ rinforza le radici per me.

(Šulgi P ll. 15-17)

28 Si segue qui nella traduzione del testo Klein (1981: 37) e commento alla linea 17 (ivi: 39). 


\section{Dcula ${ }^{23}$}

Vol 21, No 23 (July 2020) • DOI: 10.12977/ocula2020-27

Ludovica Bertolini | "Lasciate che erigano il mio letto fiorito!". Elementi vegetali nella letteratura sumerica paleo-babilonese

Nell'affermazione di Ninsumun sembra potersi leggere un accenno ai due vettori di comunicazione che il re, attraverso la sua persona, attiva tra il piano divino e quello umano. L'atto di rompere il terreno per piantare solide radici per la dea, oltre a sottolineare che Šulgi si sta costruendo un regno saldo con forti fondamenta, indica che sta ancorando il proprio operato all'ambito umano. Specificare che lo faccia 'per' la dea manifesta anche che c'è un movimento dal piano divino verso quello umano. Viceversa allungare i rami, vuol dire non solo espandere il proprio potere in un ipotetico piano orizzontale, ma anche estenderli verso l'alto, verso il cielo delle divinità e quindi lungo un vettore verticale.

Sempre più sviluppata appare la medesima immagine in Išme-Dagan ${ }^{29}$ $A+V$ (ETCSL 2.5.4.1) dove il movimento progressivo del re verso l'alto è reso esplicito nell' espressione:

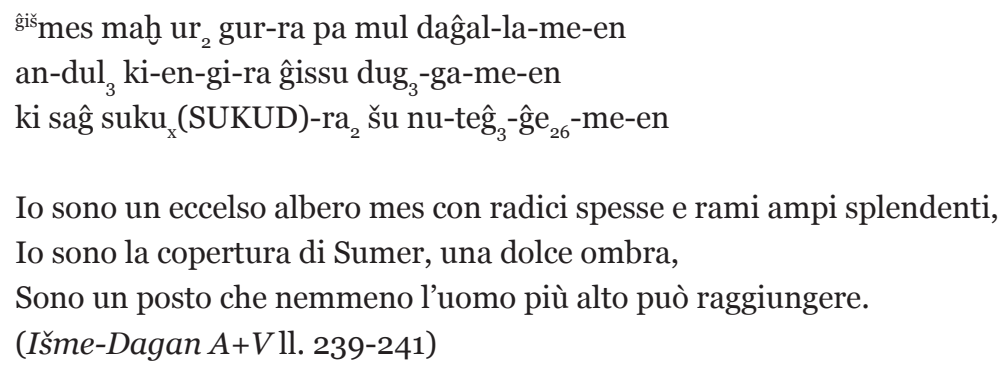

Questa definizione che il sovrano dà di se stesso è una summa delle precedenti metafore utilizzate per Ur-Namma e Šulgi. Alla linea 239 si possono individuare il tema della meraviglia, racchiuso nel termine mah, e quello della vettorialità verticale, attraverso l'utilizzo delle parti distali della pianta $\left(\mathrm{ur}_{2}\right.$; pa), che permette al sovrano di unire due piani cosmici (radici=sotto=piano umano; rami=sopra=piano divino). Alla linea 240, invece, compare l'idea di protezione e di sollievo che il sovrano offre a chi si rivolga a lui e chi si affidi al suo comando (ĝissu dug ${ }_{3}$-ga). Infine, alla linea 241 vi è un'ulteriore riproposizione della vettorialità che collega il re alle divinità, ma si introduce anche l'aspetto di unicità che caratterizza il sovrano dagli uomini comuni: egli è inarrivabile, non conosce paragone, poiché appare proiettato verso una dimensione non raggiungibile da altri uomini.

In questo senso, va intesa anche la linea 519 di Enmerkar e il signore di Aratta in cui il mitico sovrano di Uruk è paragonato ad un alto albero mes e subito dopo associato al dio Enlil, nominato qui nelle vesti di 'padre' divino del sovrano mitico (lugal-ĝu ${ }_{10}\left[{ }^{\text {ğišmes] }}\right.$ gal dumu den-lil ${ }_{2}-\mathrm{la}_{2}-\mathrm{ke}_{4}$ ). È possibile supporre che qui l'immagine dell'albero serva ad introdurre il rapporto familiare, estremamente diretto, con una divinità; la metafora servirebbe allora ad indicare l'eccezionalità del caso di Enmerkar, a elevarlo sopra agli altri uomini e a connetterlo al cielo degli dei. Si noti, inoltre, che uno dei temi centrali in questa composizione è l'affermazione da parte sia di Enmerkar che del signore di Aratta di possedere il favore di Inanna, della quale entrambi pretendono di

29 Sovrano appartenente alla Dinastia di Isin (2017-1794 a.C.). 


\section{Dcula ${ }^{23}$}

Vol 21, No 23 (July 2020) • DOI: 10.12977/ocula2020-27

Ludovica Bertolini | "Lasciate che erigano il mio letto fiorito!". Elementi vegetali nella letteratura sumerica paleo-babilonese

essere il favorito. Sarà poi chiaro nel finale che la disputa può essere vinta solo dal re di Uruk.

Fino ad ora si è parlato di come sovrani storici e mitici siano associati all'albero mes, colui che però più di tutti sembra meritare tale appellativo è proprio lo sposo di Inanna, Dumuzi. Seppur associato a tale specie solo in due canti, il già citato $D I B 29$ e $D I F$, il giovane dio è colui che meglio di tutti sembra poter incarnare gli attributi di questo albero. In $D I F$, la dea Inanna sembra compiere degli atti rituali che prevedono libagioni o aspersioni d'acqua che coinvolgono il suo sposo, qui chiamato Amaušumgalanna. Questo viene associato all'albero per mezzo di similitudini (ll. 14, 16, 31, 36) e metafore (ll. 10, 12, 25), creando un effetto di identificazione tale che non si riesce a distinguere se l'atto rituale della dea venga eseguito in presenza della pianta o dello stesso giovane.

[... A] [da]- [mul-mul] mes-ĝ $\mathrm{gu}_{10}$ pa da-e ${ }_{3}$

$\left[\mathrm{e}_{2}\right]\left[{ }^{\mathrm{d}} \mathrm{mu}\right]-\left[\mathrm{ull}-\mathrm{lil}_{2}-\mathrm{la}_{2}-\mathrm{ke}_{4}\right.$ a da-a $\hat{\mathrm{g}}_{2}-\mathrm{su}_{3}$

$[\mathrm{x} \mathrm{x}] \mathrm{x}$ a da-mul-mul mes-ğu ${ }_{10}$ pa da-an-e ${ }_{3}$

$[\mathrm{x} \mathrm{x}]\left[\mathrm{e}_{2}\right]$-kur-ra a da-a $\hat{\mathrm{g}}_{2}-\mathrm{su}_{3}$

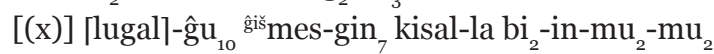

$\left[\mathrm{e}_{2}\right][\mathrm{d}][\mathrm{mu}]-$ ul-lil $\mathrm{l}_{2}-\mathrm{la}_{2}-\mathrm{ke}_{4}$ a bi $\mathrm{i}_{2}-\mathrm{in}-\mathrm{su}_{3}$

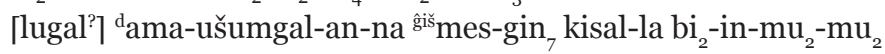

Farò risplendere [...], il mio mes, farò germogliare i rami,

$\mathrm{Nel}$ [tempio] di Enlil, spargerò acqua,

Farò splendere [...], il mio albero mes, farò germogliare i rami

[...] nell'Ekur spargerò acqua,

Il mio [...] re, come un albero mes farò crescere nel cortile,

Nel [tempio di En]lil spargerò acqua,

Il re Amaušumgalanna, come un albero mes, farò crescere nel cortile.

(DI Fll. 10-16)

Amaušumgalanna, ossia Dumuzi, nei panni del consorte di Inanna - e quindi di sovrano prototipico scelto dalla stessa dea per governare al suo fianco - è indubbiamente il tenor di queste figure retoriche e concettuali. Egli è un albero che necessita della garanzia sacrale della dea per potersi affermare (nel testo definito 'crescere'), per poter accrescere il proprio potere e la propria influenza (da cui l'immagine dei rami che germogliano e si irradiano). L'intervento della dea mette in chiaro che Dumuzi acquisisce il proprio potere per volere di Inanna: vi è un'azione di tutela che questa mette in atto per garantire all'amato legittimità di governo. In tal caso è Inanna a curarsi del suo amato come farebbe di un giardino. La menzione dell'acqua non allude, come ci si aspetterebbe dai canti d'amore, all'inseminazione della dea e, di conseguenza, del terreno. Il noto rapporto tra 'acqua' e 'liquido seminale ${ }^{30}$ non assume qui

30 Entrambi i termini si scrivono in sumerico con il segno A e spesso è solo il contesto ad indicare quale dei due significati venga chiamato in causa dal testo. 


\section{Dcula ${ }^{23}$}

Vol 21, No 23 (July 2020) • DOI: 10.12977/ocula2020-27

Ludovica Bertolini | "Lasciate che erigano il mio letto fiorito!". Elementi vegetali nella letteratura sumerica paleo-babilonese

lo stesso significato che ci si potrebbe attendere ad esempio nei miti cosmogonici riguardanti Enki; ${ }^{31}$ se si volesse vedere un'allusione nel testo all'unione sessuale dei due amanti, si dovrebbe comunque tenere presente quali siano i giochi di forza presenti nel canto e, pertanto, ricordare che la tutela del sovrano, qui nelle vesti di Dumuzi, è demandata alla sposa divina.

\subsection{Ildag}

Dell'albero conosciuto come ildag, probabilmente da riconoscersi nel pioppo, si hanno soltanto cinque attestazioni, di cui due peraltro fortemente lacunose e pertanto impossibili da inquadrare in un contesto. Tre sono le linee che verranno di seguito analizzate: Šulgi $D$ 1. 32, Šulgi $Z$ ii 15 ed un Inno a Inanna (ETCSL 4.7.a).

La prima precede una delle attestazioni cui si è avuto modo di accennare brevemente sopra, parlando del termine gurun. L'albero ildag, infatti, è menzionato in questo caso assieme ad altri elementi vegetali, che concorrono a delineare gli aspetti salienti del personaggio di Šulgi:

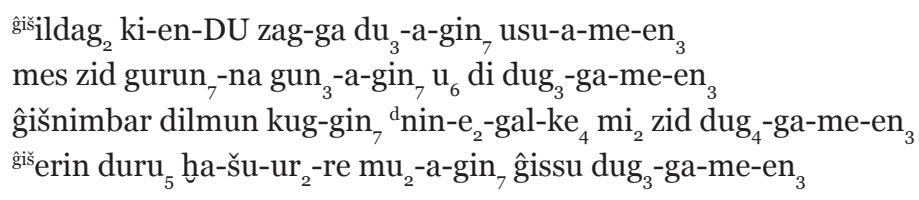

Sei forte come un albero ildag che sta eretto sul fianco del canale, Sei una dolce visione come un albero mes vitale, carico di frutti colorati, Ninegalla ti favorisce come una sacra palma da dattero di Dilmun, Sei una piacevole ombra come un cedro bagnato che cresce tra gli hašur.

(Šulgi D ll. 32-35)

Come si era visto in Išme-Dagan $A+V$, gli attributi di un giusto sovrano possono essere resi in letteratura attraverso una complessa allegoria che associ il re (quello che chiameremmo tenor) e il mondo vegetale (corrispondente al vehicle); le singole qualità di Šulgi, allora, verranno tradotte concettualmente in una serie di immagini appartenenti ad un diverso ambito, in questo caso quello floristico.

Questa volta i tratti evidenziati sono forza, fermezza o solidità, rapporto diretto con una fonte di tutela, capacità di instillare meraviglia, desiderabilità ed infine abilità di garantire protezione e beneficio per chiunque si rivolga alla figura del sovrano. Si nota immediatamente come la maggior parte delle qualità siano valide per entrambi. Ciò indica che l'ideologia regale è sostanzialmente simile sotto i due sovrani, ma anche che la struttura culturale che sottostà ai due testi è in parte sovrapponibile. In entrambi i casi l'albero mes è menzionato per fare riferimento allo splendore emanato dalla figura del re, e tutti e due i testi fanno riferimento all'ombra riflessa dalla chioma dell'albero

31 Per una discussione su tali composizioni si veda il lavoro di Leick (1994: 21-29). 


\section{Dcula ${ }^{23}$}

Vol 21, No 23 (July 2020) • DOI: 10.12977/ocula2020-27

Ludovica Bertolini | "Lasciate che erigano il mio letto fiorito!”. Elementi vegetali nella letteratura sumerica paleo-babilonese

(il sovrano) sulle sue genti. Di nuovo in Šulgi $D$ c'è la menzione dell'albero ildag, citato per la sua robustezza, grazie alla quale egli troneggia sulle rive del canale. Lo stesso cenno alla prossimità dell'albero al corso d'acqua ritengo sia un modo per riferirsi al favore divino di cui il sovrano gode. In altre parole, l'albero sarebbe qui rappresentato nelle immediate vicinanze della fonte di vita che lo sostiene, alludendo allo speciale rapporto che lega Šulgi a Inanna, che gli fa da garante. La dea è nominata infatti alla linea 34 proprio in merito alla benevolenza che mostra di riservare al re di Ur.

Sempre a Šulgi è da ascrivere la seconda menzione dell'ildag qui analizzata (Šulgi $Z$ ii 15): si tratta di una richiesta avanzata dal re a Inanna, appellata qui $\operatorname{nin}_{9}-\hat{g}_{10}$, 'sorella mia'. Il sovrano confessa di voler raggiungere alcune piante o orti in compagnia della dea, dove potrà trascorrere del tempo con lei, seducendola sotto le fronde degli alberi. Tra le specie enumerate vi è proprio l'ildag. Il passaggio, merita di essere menzionato proprio perché qui l'ildag viene nominato assieme al corso d'acqua. In questo caso, il riferimento potrebbe contenere un secondo livello interpretativo e assumere toni più licenziosi. In questo preciso contesto, infatti, il tema erotico sembra prevalere sugli intenti propagandistici che invece accentuano i tratti regali in Šulgi $D$.

Seppur in modo più sfumato, anche l'Inno a Inanna mira a esaltare la desiderabilità del pioppo invece che la sua durezza. Nel testo in questione, in una sezione sfortunatamente alquanto lacunosa, Inanna, parlando del suo rapporto con l'amato, definisce Dumuzi come un albero ildag lussureggiante che troneggia (gub, lett. 'sta') e riempie il cuore della dea di attrattiva (ll. 46-47).

\section{5. Ĝišninbar}

Il termine indica la famosa palma da dattero, pianta di estrema importanza nell'ambito della coltivazione di giardini ed orti in Mesopotamia. Contrariamente a quanto ci si aspetterebbe, però, il numero di attestazioni di questa specie nella letteratura di carattere amoroso è limitato. Il termine può comparire in combinazione con altri elementi vegetali; è questo ad esempio il caso di Šulgi $D$ ll. 34, 56 (paragrafo 3.4). Le fonti rimanenti sono, in realtà, meno interessanti. Una di queste, contenuta in Šulgi $N$, nonostante presenti il tema dell'irraggiamento dei rami dell'albero, come altri testi già citati, si riferisce al figlio del sovrano e non a lui:

[mu]-nim-mar-zu' $\mathrm{pa} \mathrm{la}_{2}-\mathrm{la}_{2}-\mathrm{de}_{3}$

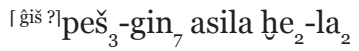

Possa la tua palma da dattero estendere i suoi rami,

Possa diffondere gioia come un albero di fichi.

(Šulgi N l. 93)

In questa benedizione che la madre pone alla fine della ninna nanna per il principe in fasce, la prima augura al futuro re di poter crescere e aumentare il

32 Si ha qui la forma emesal. 


\section{Dcula ${ }^{23}$}

Vol 21, No 23 (July 2020) • DOI: 10.12977/ocula2020-27

Ludovica Bertolini | "Lasciate che erigano il mio letto fiorito!”. Elementi vegetali nella letteratura sumerica paleo-babilonese

proprio potere sul Paese come farebbe una palma con la sua chioma, e di essere una fonte di sollievo e letizia - e quindi indirettamente di prosperità - per le sue genti, come lo sarebbe un fico che delizia chi ne assaggi i frutti.

La palma compare due volte nel cosiddetto ciclo di Dumuzi-Inanna. In DI Y, Inanna descrive l'amato come colui che si distingue (lett. 'è perfetto') nell'assemblea grazie alla sua capigliatura, somigliante alle fronde della palma da dattero. Se si tratti di una metafora o se il brano vada inteso in senso letterale e quindi ci si stia riferendo davvero alla capigliatura del dio che ricorda le fronde della palma è difficile da accertare. Sempre riferita a Dumuzi è la linea 10 di Manchester Tammuz, ${ }^{33}$ in cui Inanna si riferisce al dio come la palma da dattero del palazzo, forse alludendo alla sua bellezza o paragonandolo ai giardini che si pensa potessero ornare alcuni complessi, tra cui anche templi e palazzi (cfr. Besnier 2004). Differente invece il cenno al ĝišnimbar in $D I W$. In questo testo, vengono elencati beni tipici del mondo agro-pastorale, che serviranno a sfamare il gregge di Dumuzi, probabile metafora dietro cui può vedersi un riferimento alle genti che confidano nella figura del re. Sono convinta che, in questo caso, la presenza del gišninbar, inserito in un elenco così vasto e variegato, debba essere considerato come un elemento che assume più importanza nel contesto generale rispetto a quello che il termine stesso rappresenta. In altre parole, è l'insieme di tutte le parti ciò che interessa allo scriba, non la singola pianta.

\subsection{Erin}

Il cedro costituisce un albero importante nella tradizione letteraria in sumerico. Esso è al centro delle vicende epiche del sovrano della prima dinastia di Uruk, Gilgameš, nella famosa impresa che lo vede fronteggiare Huwawa, il guardiano della foresta dei cedri. La fragranza del cedro sembra essere un incenso molto diffuso all'interno degli edifici templari (Enlil e Sud l. 148, ETCSL 1.2.2; Cilindri di Gudea 1. 205, 356, 510, 558,), ma è anche noto l'uso del legno di questo albero per costruzioni importanti come templi o palazzi (alcuni esempi desunti dalla letteratura possono essere: La maledizione di Agade 1. 134, ETCSL 2.1.5; Cilindri di Gudea 1. 308, 596-597, 722; Lamentazione per Sumer e Ur 1. 420 ETCSL 2.2.3). Nel novero delle composizioni analizzate nel presente studio, il cedro è nominato quattro volte. Due sono state già citate: si tratta infatti di Šulgi $D$ l. 35, in cui compare l'immagine dell'ombra gettata dalle fronde del cedro - rendendo ben chiaro quale sia l'aspetto del sovrano che in questo frangente la composizione intende sottolineare ed elogiare - $\mathrm{e}$ Šulgi $P$ che paragona il sovrano ad un cedro succoso che troneggia tra i meli. Non ancora introdotte nei precedenti paragrafi sono le attestazioni di erin in Iddin-Dagan ${ }^{34} A$ (ETCSL 2.5.3.1) alle linee 178 e 186: (1992).

33 Il testo non compare nel catalogo on-line di ETCSL. Per l'edizione si veda Alster

34 Predecessore di Išme-Dagan. 


\section{Deula ${ }^{23}$}

Vol 21, No 23 (July 2020) • DOI: 10.12977/ocula2020-27

Ludovica Bertolini | "Lasciate che erigano il mio letto fiorito!". Elementi vegetali nella letteratura sumerica paleo-babilonese

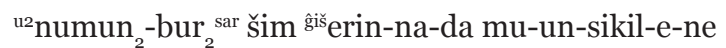

nin-ĝgu $u_{10}$ ki-nu ${ }_{2}-b i-s ̌ e_{3}$ mu-na-ab-ĝga ${ }_{2}-\hat{g a}_{2}-n e$

Erba alfalfa con fragranza di cedro hanno purificato La mia signora, sul suo letto per lei lo hanno posto. (Iddin-Dagan A l.178-179)

$\mathrm{i}_{3}$ šim firišerin-na ki am $_{3}$-sud-e

Ella (Inanna) cosparge il pavimento con olio e fragranza di cedro (Iddin-Dagan A l. 186)

Ci troviamo di fronte a uno dei passi relativi al cosiddetto matrimonio sacro. Il sovrano Iddin-Dagan, nel suo inno viene rappresentato nei momenti di intimità trascorsi assieme alla dea. Il punto focale di questi canti è costituito generalmente da due azioni rituali: la preparazione della dea $^{35}$ (al centro di molti canti del cosiddetto ciclo di Dumuzi-Inanna) e l'ornamento del letto con annessa consumazione dell'atto sessuale tra la dea e il suo amato (in rari casi, come in Iddin-Dagan A, viene fatto accenno alla preparazione del letto sul quale i due protagonisti trascorreranno insieme la notte). È qui fondamentale notare come il cedro ricopra in questo testo un ruolo fondamentale nel processo di purificazione del giaciglio sacro. Che il cedro venga considerato un albero dai connotati particolari si può intuire dal fatto che nella tradizione letteraria esso fosse sorvegliato da un personaggio mitico incaricato dallo stesso dio Enlil di proteggere la foresta. L'aroma del cedro viene chiaramente descritto nei $\mathrm{Ci}$ lindri di Gudea l. 205 come 'fragranza degli dei' (šim erin ir-sim diĝir-ra-kam), e questo pare concordare perfettamente con il ruolo purificatore della pianta in contesti rituali. Tuttavia, non sembra che l'erin sia l'unico elemento vegetale che possa assolvere un tale compito. In $D I T$ l. 41 il letto della dea viene cosparso di 'erbe (simili a) lapislazzuli lucenti' $\left(\mathrm{u}_{2}\right.$-za-gin ${ }_{3}-\mathrm{dur}_{5}-\mathrm{ru}$ ), invece in $D I Z$ il letto è probabilmente ricoperto di fiori (mu-na ${ }_{2}$-gi-rin-e). In $D I D 1$ il dio Gibil è il responsabile delle cerimonie di purificazione del letto della dea. Il fine di tali atti è di permettere il corretto svolgimento dei rituali relativi allo status di 'essere regina' ${ }^{6}$ (nam-ga-ša-an) e secondo le linee 11-12 egli avrebbe compiuto tali atti rituali con l'aiuto dell'erba alfalfa e delle canne-ušub. Non è possibile purtroppo riuscire a ricostruire un rituale preciso basandosi su questi testi e non è nemmeno certo che ne esistesse uno solo. Il fatto che però queste particolari erbe siano nominate in contesti così speciali, deve comunque significare che fosse loro attribuito un enorme valore sacrale e che l'elemento vegetale dovesse avere un valore ai fini della buona riuscita della performance.

35 Per una trattazione sul tema si veda Verderame (2009).

36 La difficoltà di tradurre in italiano il termine sta nella mancanza, nella lingua italiana, di sostantivi distinti che permettano di esprimere concetti astratti come "regalità o status di re" e "status di regina" mantenendone i connotati di genere. Con nam-ga-ša-an, infatti, si intende l'essenza dell'essere regina. 


\section{Deula ${ }^{23}$}

Vol 21, No 23 (July 2020) • DOI: 10.12977/ocula2020-27

Ludovica Bertolini | "Lasciate che erigano il mio letto fiorito!”. Elementi vegetali nella letteratura sumerica paleo-babilonese

\subsection{Nâga}

Una pianta conosciuta con il nome di naĝa (emesal: nama) appare nei componimenti di carattere amoroso. Il contesto ricorrente è quello di atti rituali relativi all'ingresso in casa dei suoceri da parte del dio Dumuzi. L'azione rituale che richiede l'utilizzo di questa pianta è la lustrazione del corpo della dea Inanna. In alcuni testi del ciclo, la dea prima di unirsi al suo sposo, esegue una serie di gesti - descritti generalmente in modo simile nelle diverse composizioni - che comprendono: il lavaggio del corpo con acqua e potassa derivata dalla lavorazione dell'erba naĝa, l'uso di oli per il corpo, l'acconciatura dei capelli, la vestizione e infine l'assunzione dei simboli della divinità e del comando tipici della dea. Tali atti non compaiono sempre contemporaneamente nei vari componimenti e il loro ordine può variare leggermente, ma è chiaro che nei testi si alluda ad un medesimo contesto rituale.

Le attestazioni qui evidenziate inerenti l'uso dell'erba naĝa, o della potassa da essa ricavata, sono in tutto nove, suddivise in sei testi.

In $D I C$ si può trovare una descrizione piuttosto dettagliata del processo di preparazione di Inanna. Alla domanda dell'amato su cosa stia facendo in casa, Inanna risponde elencando le azioni che ha compiuto prima che egli arrivasse:

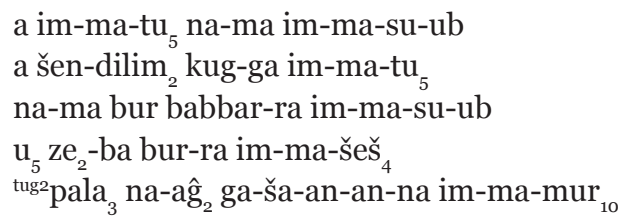

Mi sono lavata con acqua, mi sono frizionata con erba naĝa (potassa)

Mi sono lavata con acqua della pura brocca-šendili

Mi sono frizionata con erba naĝa (potassa) della ciotola bianca

Mi sono unta con olio fine della ciotola

Ho indossato l'abito della regalità ('essere regina')

(DI C ll. 3-7)

È probabile che l'erba venisse processata prima dell'uso. Dalla lavorazione di questa materia prima si doveva ricavare, verosimilmente, una sorta di pasta da riporre in uno speciale contenitore. Quest'ultimo avrebbe potuto far parte degli arredi sacri del tempio, di modo che l'erba potesse essere utilizzata quando se ne fosse ravvisato il bisogno. Il termine bur richiama di per sé l'ambito sacro. La traduzione di questo sostantivo varia da 'offerta', 'sacrificio' a 'ciotola (in pietra)'. L'aggettivo babbar potrebbe riferirsi al colore della pietra utilizzata, ma anche alla natura splendente del contenitore e perciò legata ad un ambito ritualmente connotato. Purtroppo mancano informazioni più approfondite su come tale erba venisse trattata in queste occasioni speciali.

Analoga a quella del brano precedente è la descrizione della scena in DI Pl. 28 , in cui ricompare sia il bacino šen che la veste pala. L'uso di acqua e potassa segue il medesimo ordine, anche se qui non viene ripetuto il gesto nella linea successiva e manca il contenitore in cui l'erba naĝa è riposta: 


\section{Ocula ${ }^{23}$}

Vol 21, No 23 (July 2020) • DOI: 10.12977/ocula2020-27

Ludovica Bertolini | "Lasciate che erigano il mio letto fiorito!". Elementi vegetali nella letteratura sumerica paleo-babilonese

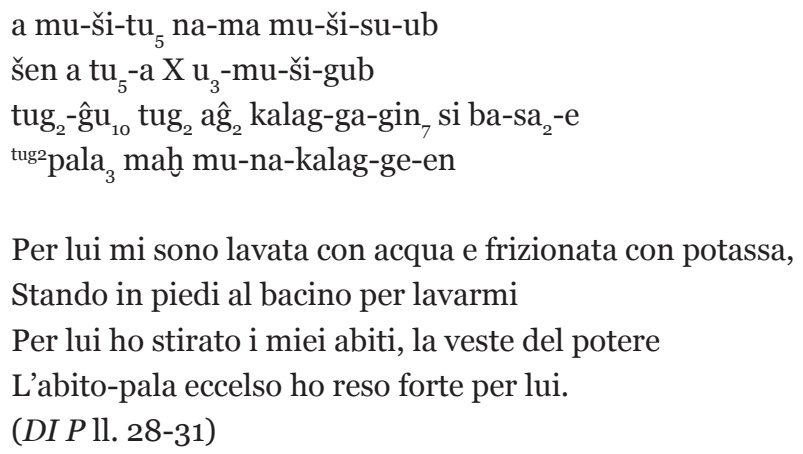

Il fatto che non vengano date maggiori informazioni su questi atti, potrebbe suggerire che dovessero essere più o meno noti allo scriba, tanto da non richiedere ulteriori spiegazioni. La potassa, inoltre, doveva essere uno strumento piuttosto diffuso. Appare sia in ambito rituale (cfr. Couto-Ferreira 2017: 104, n. 28) che nell'uso quotidiano, almeno per quanto concerne alcune specifiche categorie di individui. Sappiamo, infatti, che in alcuni casi questa particolare erba veniva consegnata, assieme alle razioni di cibo, ai messaggeri (Notizia 2009: 24).

Pressoché identico al brano contenuto in $D I C$ è ancora un passo inerente la preparazione di Inanna: questa volta le linee in questione appartengono al canto, purtroppo fortemente lacunoso, DI E1. La dea, che non viene qui nominata nelle parti preservatesi, ma riconoscibile anche per la presenza nella scena della madre di Dumuzi, Duttur, dopo aver partecipato ad una processione rituale sembra dedicarsi finalmente alla solita serie di atti rituali prima di ricevere la visita della futura suocera.

Nella composizione Iddin-Dagan A troviamo l'unica attestazione della preparazione della dea all'unione con il sovrano che comprenda l'erba naĝa:

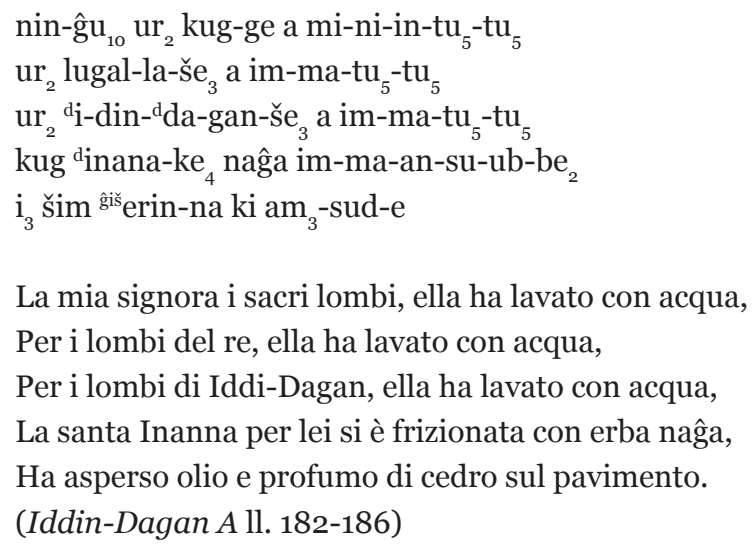

La formula in questo inno è stata modificata, mentre l'ordine degli eventi è stato mantenuto. Tuttavia, si è deciso di dare maggiore rilievo alla coporalita e alla fisicità della dea ponendo l'accento sulla zona dei fianchi o del ventre per rendere più esplicito il ruolo del sovrano nel rituale. Il parallelismo tra il 


\section{Dcula ${ }^{23}$}

Vol 21, No 23 (July 2020) • DOI: 10.12977/ocula2020-27

Ludovica Bertolini | "Lasciate che erigano il mio letto fiorito!". Elementi vegetali nella letteratura sumerica paleo-babilonese

termine $\mathrm{ur}_{2}$ utilizzato alla linea 182 per indicare i fianchi della dea e alle linee 183-184 per riferirsi a quelli del re serve a legare ancor più intimamente i due personaggi. Come si può vedere il rituale, in sé, non è cambiato; la dea compie le medesime azioni che avrebbe eseguito se si fosse trattato di Dumuzi. L'identificazione funzionale tra sovrano e sposo divino della dea è pressoché assoluta, come suggerisce la menzione di Amaušumgalanna alla linea 189.

In un passo dei Cilindri di Gudea, il re riporta di aver installato Kinda-zid, un'entità divina poco conosciuta, nel tempio Eduga per occuparsi del ben più importante dio Ningirsu. Tra i compiti di Kinda-zid vi sono quelli di lavare Ningirsu con acqua e di frizionarlo con l'erba naĝa per poi cospargerlo con oli. È probabile che, seppur ad un livello mitico, Gudea stia affidando a Kinda-zid la cura della statua del dio poliade di Girsu. L'effige, come era prassi rituale, doveva essere lavata, 'nutrita' e fatta riposare come se si trattasse del dio in persona. La potassa infine può servire anche nei rituali di purificazione dei templi, come suggerisce la linea 6 (kur ki sikil-la naĝa dub $_{2}$ dug $_{4}$-ga) dell'Inno ai templi (ETCSL 4.80.1).

Le proprietà purificatrici riconosciute a questa erba dovevano essere condivise anche da altre specie. Vi sono infatti alcune piante, conosciute dai testi in questione, che assumono l'aggettivo sikil: un esempio può essere l'innuš (DI R i 25, iii 14), o l'ilanum su cui l'eroe di Uruk, Lugalbanda, si stende per ricevere un sogno che possa aiutarlo ad entrare in contatto con la divinità ( $L u$ galbanda e la caverna 1. 337, ETCSL 1.8.2.1).

\section{Conclusioni}

La funzione degli elementi vegetali all'interno della documentazione letteraria non è rigida e costante, ma ha invece una certa fluidità e capacità di adattamento alle necessità del testo. Una stessa pianta può comparire in un caso come vehicle all'interno di metafore atte a descrivere personaggi o ambientazioni e, in un altro, come uno degli elementi necessari allo svolgimento di atti rituali. Nel primo caso, si è visto come le caratteristiche qualitative di alberi e piante ben si prestino a veicolare concetti quali grandezza, forza ed imbattibilità nel caso del sovrano, mentre quanto vengono associate alla dea Inanna evidenziano aspetti come dolcezza, desiderabilità e sensualità. Si è notato, inoltre, che uno stesso termine può riferirsi indistintamente ad entrambi gli amanti, che pure condividono tratti in comune come la capacità di suscitare meraviglia o attrazione. Indicativo è anche il fatto che non vi sia differenza nel modo in cui le specie vegetali tendono a descrivere la figura del sovrano rispetto a quella del dio Dumuzi. In tal modo, di fatto si rafforza quel legame che i sovrani intendono mantenere saldamente con la sfera divina, per garantire un supporto ideologico forte al potere che essi esercitano. L'elemento vegetale, quando compare nella descrizione di paesaggi o ambientazioni, tende a sottolineare quei caratteri dell'ecosistema che più si accordano con la situazione narrata nel componimento. Per questo motivo, i luoghi di incontro tra innamorati sono rappresentati carichi di frutti e di alberi frondosi che offrono riparo, in modo da celebrare l'abbondanza del Paese. Più che di 
fertilità vera e propria, allora, credo si debba parlare di prosperità. ${ }^{37}$ L'unione del sovrano (o di Dumuzi) con la dea, nella letteratura di questo periodo, non suggerisce che Inanna sia primariamente collegata al concetto di sessualità riproduttiva, come si può evincere dalla mancata menzione di una progenie..$^{38}$ In tal senso non si dimentichi il legame che avvince Inanna a figure che sono use a rapporti non finalizzati alla procreazione come nel caso delle prostitute (vedi Inanna H, ETCSL 4.7.8). Il sovrano, pastore che cura il proprio gregge, si assicura l'amore ed il sostegno della dea per garantire al suo regno tutto ciò di cui ha bisogno. Lo scambio avviene secondo entrambe le direttive, dal piano divino a quello umano e viceversa. Questa complessa operazione può essere resa in letteratura attraverso la metafora dell'albero, che affonda le radici nel terreno e protende i suoi rami verso il cielo. Allo stesso modo, infatti, il sovrano rinsalda il proprio governo nell'ambito terreno e si cura delle divinità con rituali che dimostrino e rinnovino sacralmente il suo impegno a servire gli dei.

L'elemento vegetale, con il quale l'uomo mesopotamico è in contatto pressoché ogni giorno, costituisce un mezzo perfetto per dare forma a concetti che altrimenti non sarebbero facilmente descrivibili. Aspetti dell'emotività difficili da esprimere attraverso concetti astratti, o semplicemente non tangibili ed invisibili, come possono essere l'impulso sessuale, l'amore, il benessere, il vigore, possono trovare nel mondo fenomenico un buon mezzo espressivo tramite la costruzione di figure retoriche che affondino in un background culturale noto a chi deve interpretarle.

Gli elementi naturali che richiamano l'ambito della prosperità, della lussuria e del piacere in generale come fiori o erbe profumate, alludono da una parte all'ambiente naturale che fa da sfondo alle vicende d'amore di Dumuzi ed Inanna, e dall'altra creano un legame con il personaggio di Dumuzi, che nella steppa ha la sua sfera di influenza. I fiori che ornano e purificano il letto all'interno del tempio richiamano indirettamente quelli su cui la dea e il suo amato si stendono nell'intimità del giardino. Il cosmos controllato di quest'ultimo è esso stesso un simbolo dell'azione ordinatrice del sovrano garantita dalla tutela divina, che ne rinnova l'azione cosmogonica. Non si deve però dimenticare che alcune di queste piante assumono anche un differente ruolo nei rituali presentati. La presenza della pianta naĝa, o di altre specie considerate in qualche modo sacre o pure, come sembra essere il caso della pianta innuš o ilanum, ci ricorda che gli elementi vegetali hanno anche una propria valenza intrinseca. L'unicità ed eccezionalità del rapporto tra Inanna ed il suo sposo richiede che i due si presentino puri e nel pieno delle proprie prerogative divine per far sì che il rituale avvenga correttamente; da qui l'interesse nelle composizioni per una sequenza di azioni che potrebbero sembrare ad un primo sguardo delle semplici formalità. Il fatto che, nei vari testi, ogni singolo gesto venga presentato con una struttura più o meno formulare serve a sot-

37 Già Vanstiphout (1984: 226) aveva avanzato delle riserve in tal senso.

38 Si sa che per alcune tradizioni a carattere locale il dio Šara viene considerato il figlio della dea (si veda Black e Green 1992), tuttavia si tratta di casi isolati. 


\section{Ocula ${ }^{23}$}

Vol 21, No 23 (July 2020) • DOI: 10.12977/ocula2020-27

Ludovica Bertolini | "Lasciate che erigano il mio letto fiorito!". Elementi vegetali nella letteratura sumerica paleo-babilonese

tolinearne la solennità. Il risultato della corretta esecuzione di queste azioni sacrali, che mirano a rinnovare l'atto fondativo del tempo del mito, è descritta approfonditamente nelle composizioni. In tal senso vanno interpretate le benedizioni fatte dalla dea all'amato, in cui compaiono lunghi elenchi di specie vegetali. Queste devono essere considerate nel loro insieme, come espressione di una totalità e completezza che permette al Paese di ricevere, per mezzo del sovrano, tutto ciò di cui abbia bisogno.

In definitiva, nonostante la diversificata varietà di usi che possano avere $\mathrm{i}$ singoli elementi vegetali all'interno del corpus letterario e tradizionale sumerico inerente le figure di Inanna e del suo sposo, un ruolo che tutti essi condividono è la creazione di un immaginario di benessere che solo il re che si affidi alla sfera divina e ne riceva la benedizione è in grado di garantire al regno.

\section{Bibliografia}

Alster, Bendt

1971 "On the Sumerian Lullaby", RA, 65, 1971, pp. 170-171.

1992 “The Manchester Tammuz", ASJ, 14, 1992, pp. 1-46.

Besnier, Marie-Françoise

2002 “Temptation's Garden: The Gardener, a Mediator Who Plays an Ambiguous Part", in Parpola, S. e Whiting, R.M. (a cura di), Sex and Gender in the Ancient Near East. Proceedings of the 47th Rencontre Assyriologique Internationale, Helsinki, July 2-6, 2001. Part I-II. Helsinki, The Neo-Assyrian Corpus Project, pp. 59-70.

2004 "Vegetation in Mesopotamian Temple Precints: Gardens "Sacred Groves" or Potted Plants", JAC, 19, 2004, pp. 59-87.

Black, Jeremy A.; Green, Anthony

1992 Gods, Demons and Symbols of Ancient Mesopotamia. An Illustrated Dictionary. London. The Trustees of the British Museum.

Cooper, Jerrold

1989 "Enki's Member: Eros and the Irrigation in Sumerian Literature", in Behrens, H. et alii (a cura di), DUMU-E2-DUB-BA-A. Studies in Honor of Åke W. Sjöberg, Occasional Publications of the Samuel Noah Kramer Fund 11, Philadelphia, University Museum, pp. 87-89.

Couto-Ferreira, Erica M.

2017 "Let me be your canal': some thoughts on agricultural landscape and female bodies in Sumero-Akkadian sources", in Feliu, L.; Karahashi, F; Rubio, G. (a cura di), The First Ninety Years: A Sumerian Celebration in Honor of Miguel Civil, Boston / Berlin, de Gruyter, pp. 54-69.

George, Andrew

2003 The Babylonian Gilgamesh Epic. Introduction, Critical Edition and Cuneiform Texts, vol. I-II, Oxford, Oxford University Press.

Greco, Angela

2015 Garden Administration in the Girsu Province During the Neo-Sumerian Period (BPOA 12), Madrid, Consejo Superior de Investigaciones Científicas. 


\section{Ocula ${ }^{23}$}

Vol 21, No 23 (July 2020) • DOI: 10.12977/ocula2020-27

Ludovica Bertolini | "Lasciate che erigano il mio letto fiorito!". Elementi vegetali nella letteratura sumerica paleo-babilonese

Harris, Rivkah

1991 "Inanna-Ishtar as Paradox and a Coincidence of Opposites", History of Religions, 30, 1991, pp. 261-278.

Jacobsen, Thorkild

1975 "Religious Drama in Ancient Mesopotamia”, in Goedicke, H. e Roberts, J.J.H. (a cura di), Unity and Diversity: Essays in History, Literature, and Religion of Ancient Near East (JHNES 7), Baltimore/London, Johns Hopkins University Press, pp. 65-97.

Leick, Gwendolin

1994 Sex and Eroticism in Mesopotamian Literature, London/NewYork, Routledge.

Notizia, Palmiro

2009 I testi dei messaggeri da Ĝirsu-Lagaš della Terza Dinastia di Ur, Messina, Di.Sc.A.M.

Ponchia, Simonetta

2009 "Some Reflections on Metaphor, Ambiguity and Literary Tradition" in Luukko, M.; Svärd, S.; Mattila, R. (a cura di), Of God(s), Trees, Kings, and Scholars. Neo-Assyrian and Related Studies in Honour of Simo Parpola (StOr 106), Helsinki, The Neo-Assyrian Corpus Project, pp. 399-407.

Rubio, Gonzalo

2009 "Sumerian Literature", in Ehrlich, C.S. (a cura di), From an Antique Land: an Introdution to Ancient Near Eastern Literature, Lahnam, Rowman and Littlefield Publishers, pp. 11-66.

Schretter, Manfred K.

1990 Emesal-Studien. Sprach- und literaturgeschichtliche Untersuchungen zur sogenannten Frauensprache des Sumerischen (Innsbrucker Beiträge zur Kulturwissenschaft 69), Innsbruck, Verlag des Instituts für Sprachwissenschaft.

Sefati, Yitschak

1998 Love Songs in Sumerian Literature, in Bar-Ilan Studies in Near Eastern Languages and Culture. Publication of the Samuel N. Kramer Institute of Assyriology, Ramat Gan, Bar-Ilan University Press.

Sefati, Yitschak; Klein, Jacob

2012 "Two Dumuzi-Inanna Love Songs: Dumuzi-Inanna Q and an Unidentifies Song”, in Abraham, K. e Fleishman, J. (a cura di), Looking at the Ancient Near East and the Bible through the Same Eyes, Minha LeAhron: A Tribute to Aaron, Bethesda, CDL Press, pp. 309-329.

Selz, Gebhard J.

2014 "Plant Metaphors: on the Plant of Regiuvenation", in Gaspa, S. et al. (a cura di), From Source to History. Studies on Ancient Near Eastern Worlds and Beyond. Dedicated to Giovanni Battista Lanfranchi on the Occasion of His 65th Birthday on Ĝne 23, 2014 (AOAT 412), Münster, Ugaritverlag, pp. 655-667.

Vanstiphout, Herman L. J.

1984 “Inanna/Ishtar as a Figure of Controversy: Struggles of Gods", in Kippenberg, H. G.; Drijvers, H.J.W.; Kuiper, Y. (a cura di), Papers of the Groningen Work Group for the Study of the History of Religions (Religion and Reason, 31), Berlin/New York/Amsterdam, Walter de Gruyter/Mouton Publishers, pp. 225-238. 


\section{Deula ${ }^{23}$}

Vol 21, No 23 (July 2020) • DOI: 10.12977/ocula2020-27

Ludovica Bertolini | "Lasciate che erigano il mio letto fiorito!”. Elementi vegetali nella letteratura sumerica paleo-babilonese

Verderame, Lorenzo

2009 "La vestizione di Inanna" in Botta, S. (a cura di), Abiti, corpi, identità. Significati e valenze profonde del vestire, Firenze, Società Editrice Fiorentina, pp. 63-73.

Westenholz, Joan G.

1992 "Metaphorical Language in the Poetry of Love in the Ancient Near East", in Charpin, D. e Joannès, F. (a cura di), La circulation des biens, des personnes et des idées dans le Proçhe-Orient ancien. Actes de la XXXVIIIe Rencontre Assyriologique Internationale, Paris, 8-10 juillet, 1991, Paris, Editions Recherche sur les civilisations, pp. 381-387.

Whittaker, Gordon

2002 "Linguistic Anthropology and the Study of Emesal as (a) Women's Language", in Parpola, S. e Whiting, R.M. (a cura di), Sex and Gender in the Ancient Near East. Proceedings of the 47th Rencontre Assyriologique Internationale, Helsinki, July 2-6, 2001. Part I-II. Helsinki, The Neo-Assyrian Corpus Project, pp. 633-644.

Wilcke, Claus

1987 A Riding Tooth: Metaphor, Metonymy and Synecdoche, Quick and Frozen in Everyday Language, in Mindlin, M. et al. (a cura di), Figurative Language in the Ancient Near East, London, University of london, pp. 77-102.

Zisa, Gioele

[in stampa] "Il corpo sessuato delle dee. Agricoltura, pastorizia e mondo vegetale nella Mesopotamia antica”, in Bonanno, D. e Buttitta, I.E. (a cura di), Narrazioni e rappresentazioni del sacro femminile. Atti del convegno internazionale in memoria di Giuseppe Martorana, Acta diurna, Fondazione Buttitta.

Ludovica Bertolini ha ottenuto il Dottorato di Ricerca in Storia, Antropologia, Religioni presso l'Università di Roma La Sapienza nel settembre 2019 con una tesi riguardante le divinità sumeriche Inanna e Dumuzi. Attualmente, Bertolini si sta occupando del ruolo giocato dalle metafore nella costruzione della concezione di 'spazio' all'interno della tradizione letteraria in lingua sumerica risalente al periodo paleo-babilonese. 